\title{
Allopurinol and the risk of stroke in older adults receiving medicare
}

Jasvinder A. Singh ${ }^{1,2,3,4,5^{*}}$ and Shaohua $\mathrm{Yu}^{2}$

\begin{abstract}
Background: Previous studies of allopurinol and stroke risk have provided contradictory findings, ranging from a protective effect to an increased risk. Our objective was to assess whether allopurinol use is associated with the risk of stroke in the elderly.

Methods: We used the $5 \%$ random sample of Medicare beneficiaries from 2006-2012 to study the association of new allopurinol initiation and incident stroke. We used multivariable-adjusted Cox regression models adjusted for age, gender, race, Charlson index, and cardio-protective medications (beta-blockers, ACE inhibitors, diuretics, statins) to calculate hazards ratio (HR) with $95 \%$ confidence intervals (Cl). Sensitivity analyses adjusted for coronary artery disease (CAD) risk factors including hypertension, hyperlipidemia, diabetes, and smoking instead of Charlson index.

Results: Among 28,488 eligible episodes of incident allopurinol, 2,177 ended in incident stroke (7.6 \% episodes). In multivariable-adjusted analyses, allopurinol use was associated with $9 \%$ lower hazard ratio for stoke, 0.91 (95\% Cl, 0.83 to 0.99 ). Compared to no allopurinol use, allopurinol use durations of 181 days to 2 years, $0.88(95 \% \mathrm{Cl}, 0.78$ to $0.99)$ and $>2$ years, $0.79(95 \% \mathrm{Cl}, 0.65$ to 0.96$)$ were significantly associated with lower multivariable-adjusted hazard of stroke. Sensitivity analyses adjusted for CAD risk factors confirmed these findings. In subgroup analyses, significant associations were noted between allopurinol use and the risk of ischemic stroke, 0.89 (95\% Cl, 0.81 to 0.98); associations were not significant for hemorrhagic stroke, $1.01(95 \% \mathrm{Cl}, 0.79$ to 1.29).
\end{abstract}

Conclusions: Allopurinol use is associated with lower risk of stroke overall, more specifically ischemic stroke. This association is evident after 6-months of allopurinol use, and the hazard reduction increases with longer duration of use. Future studies need to examine underlying mechanisms.

Keywords: Allopurinol, Stroke, Elderly, Medicare, Ischemic stroke, Urate-lowering therapy, Hyperuricemia, Age, Race, Gender

Abbreviations: ACE inhibitor, Angiotensin converting enzyme inhibitor; CAD, Coronary artery disease; ED, Emergency department; ICD-9-CM, International classification of diseases, ninth revision, common modification codes; COPD, Chronic obstructive pulmonary disease; NEDS, National ED sample; ULT, Urate-lowering therapy

\section{Background}

Stroke is the second leading cause of cardiovascular mortality worldwide and among the top five causes of death in the U.S. $[1,2]$. In the U.S., 6.4 million people, $2.7 \%$ of the adult population, suffered from stroke, according to the 2012 National Health Interview Survey [3]. Stroke is associated with significant disability, and a

\footnotetext{
* Correspondence: Jasvinder.md@gmail.com

${ }^{1}$ Medicine Service, Birmingham VA Medical Center, Birmingham, AL, USA

${ }^{2}$ Department of Medicine at School of Medicine, University of Alabama at Birmingham (UAB), Birmingham, AL, USA

Full list of author information is available at the end of the article
}

negative impact on function and quality of life [4-7]. The treatment for stroke has rapidly evolved over time, and outcomes continue to improve [8]. While some of the disease risk factors for stroke are well known, there is a relative lack of studies assessing whether certain commonly used medications can modify the risk of stroke.

Allopurinol is a urate-lowering therapy (ULT) that is commonly used for the treatment of hyperuricemia $[9,10]$. In addition to its urate-lowering effect related to of the inhibition of xanthine oxidase by its active metabolite, oxypurinol, recent studies have suggested other mechanisms of 
actions of allopurinol, some dependent and some independent of xanthine oxidase inhibition [11-18]. Many of these effects including improvement of endothelial function and reduction of oxidative stress [14, 19-23], reduction of glycosylated hemoglobin [14], and attenuation of intercellular adhesion molecule-1 levels [24], may potentially lower the risk of stroke. To our knowledge, it is not known whether allopurinol use is associated with a lower risk of stroke.

Previous studies of allopurinol examined a composite cardiovascular outcome (that included stroke) and provided contradictory findings, ranging from a protective effect $[25,26]$ to an increased risk [27, 28]. These contradictory findings were related at least partially to differences in patient populations (renal failure, hypertensive nephropathy, heart failure vs. gout), study design (randomized vs. observational) and covariates/ confounders adjusted in the analyses. In a 7-year follow-up of a 2-year randomized trial in patients with chronic kidney disease, adjusted for race, sex and renal function, allopurinol use was associated with lower risk of 0.43 (95\% CI, 0.21 to 0.88 ) of cardiovascular events (including CAD, cerebrovascular disease, heart failure and peripheral vascular disease) [25]. In an observational study of 187 patients with hypertensive nephropathy, allopurinol use was associated with an adjusted hazard of 0.34 $(p=0.04)$ for cardiovascular disease (CAD, heart failure and stroke) [26]. In a study of heart failure patients using Scottish database, Wei et al. reported that with a minimum follow-up of 5 years, compared with non-users, allopurinol users had a no significant difference in cardiovascular event (nonfatal myocardial infarction (MI), nonfatal stroke and cardiovascular mortality), 0.88 (95\% CI, 0.73 to 1.05) [27]. Kok et al. [28] studied $>4,000$ patients with newly diagnosed gout and no pre-existing severe form of CVD using a prevalent user design and reported that allopurinol use was associated with an increased adjusted hazard of 1.25 for cardiovascular event (hospitalization for MI, stroke, hypertension etc.).

None of the previous studies examined the association of allopurinol with stroke as an outcome in the general population. Therefore, findings can only be generalized to patient sub-populations with hypertensive nephropathy, renal failure, gout or heart failure, and then only to a cardiovascular composite outcome, that includes peripheral vascular disease, heart failure, MI, angina etc. in addition to stroke. Thus, well-designed studies examining the effect of allopurinol use on the risk of stroke are needed, especially for adults 65 years and older. This is important since $2 / 3^{\text {rd }}$ of patients with stroke in the U.S. are adults 65 years and older [29]. Therefore, our objective was to assess whether incident allopurinol use was independently associated with a reduction in the risk of incident stroke in the elderly, using a population-based approach. We hypothesized that allopurinol use and its duration of use will each be independently associated with a reduction in the risk of incident stroke in adults 65 years and older. In exploratory analyses, we examined whether stroke risk reduction with allopurinol varied by age, gender and race.

\section{Methods}

\section{Study cohort and population of interest}

We conducted a retrospective cohort study using the $5 \%$ random sample of Medicare beneficiary claims from 2006 to 2012. These data were obtained from the Centers for Medicare and Medicaid Services (CMS) Chronic Condition Data Warehouse and included beneficiaries' demographic information and insurance claims (inpatient, outpatient, skilled nursing facility, non-institutional provider, home health, hospice, durable medical equipment and prescription drugs).

Study eligibility criteria were: (1) Medicare beneficiaries who were 65 years of age or older and lived in the U.S.; (2) continuous enrollment in traditional Medicare fee-for-service and pharmacy coverage (Parts A, B and D) and non-enrollment in Medicare Advantage Plan; and (3) new treatment with allopurinol (defined below). The Institutional Review Board at the University of Alabama at Birmingham approved the study, and waived the need for patient informed consent for this database study. All investigations were conducted in conformity with ethical principles of research.

\section{Exposure definition and covariates}

Incident allopurinol use, i.e. new allopurinol use, was defined as a new filled allopurinol prescription, with no allopurinol prescription filled during a look-back baseline period of 365 days. Each day of observation within each episode was labeled as allopurinol exposed or nonexposed based upon the days' supply for allopurinol prescription in pharmacy records after the beginning of allopurinol treatment episode. We allowed up to 30 days stock carry over. Patients were considered exposed for 30 days after the end of days' supply to capture events attributable to allopurinol, after which a new continuous allopurinol exposure period started. Allopurinol use duration was categorized as none, 1 to 180 days, 181 days to 2 years, and longer than 2 years, based on clinical relevance and current use patterns of allopurinol $[9,10]$. A patient could contribute multiple allopurinol treatment episodes during different time periods.

We obtained the following covariates in the baseline period for each allopurinol treatment episode from the Medicare denominator file: age, gender, race/ethnicity, residence (Northeast, South, Midwest, West) and comorbidity scores, derived using Charlson-Romano comorbidity index 
score, a validated score for comorbidity assessment consisting of common medical comorbidities [30], adapted for claims data by Romano et al.. Comorbidities included in Charlson-Romano comorbidity index were: myocardial infarction, congestive heart failure, cerebrovascular disease, dementia, chronic pulmonary disease, connective tissue disease, peptic ulcer disease, mild liver disease, diabetes without complications, diabetes with complications, paraplegia and hemiplegia, renal disease, cancer, moderate to severe liver disease, metastatic cancer and AIDS/HIV [31]. Charlson-Romano index score is a valid comorbidity measure [32, 33].

\section{Study outcome}

Incident stroke was our study outcome of interest, defined as the first occurence of stroke during the study period after the initiation of a new allopurinol prescription. Incident stroke was identified by the presence of International Classification of Diseases, ninth revision, common modification (ICD-9-CM) codes for stroke (430.xx, 431.xx, 433.x1, 436.xx, 434.xx, except 434.x0); subgroup analyses were for hemorrhagic stroke (430.xx, 431.xx) and ischemic stroke (rest of the codes). This approach using ICD-9-CM codes is accurate for identification of cases and risk factors, and has high positive predictive value, usually exceeding $>90 \%$ [34-36]. The earliest allopurinol treatment initiation date during the study period marked the beginning of the follow-up for each treatment episode that ended on the earliest of first date of stroke, losing full Medicare coverage, the date of death, or the end of the study $(12 / 31 / 12)$.

\section{Statistical analyses}

We calculated descriptive statistics by the occurrence of stroke among allopurinol users and by allopurinol exposure among episodes with stroke. The main analysis focused on stroke (including all subtypes). We performed Cox proportional hazard regression models to assess the association between allopurinol exposure or duration of allopurinol use and incident stroke. Multivariable analysis were adjusted for age, gender, race and Charlson-Romano comorbidity score. We accounted for correlated episodes using the Huber-White "Sandwich" variance estimator and calculated robust standard errors for all estimates, since patients could possibly contribute more than one episode of new allopurinol use. We performed sensitivity analyses using Cox proportional hazard regression models to assess these associations by replacing CharlsonRomano index score with selected comorbidities, including risk factors for coronary artery disease (CAD) in one model, and replacing with peripheral vascular disease (PVD) and CAD in another separate model. We calculated hazard ratio (HR) and $95 \%$ confidence intervals (CI). We also performed subgroup analyses by assessing the relationship of allopurinol use and allopurinol use duration to each type of stroke, ischemic vs. hemorrhagic stroke.

Table 1 Demographic and clinical characteristics of episodes of incident ${ }^{a}$ allopurinol use

\begin{tabular}{|c|c|c|c|c|}
\hline & \multirow[t]{2}{*}{ All } & \multicolumn{2}{|c|}{ Incident stroke during follow up } & \multirow[t]{2}{*}{$P$-value } \\
\hline & & No & Yes & \\
\hline Total, N (episodes) & 28,488 & 26,311 & 2,177 & \\
\hline Age & $76.5(7.4)$ & $76.4(7.4)$ & $78.1(7.3)$ & $<0.0001$ \\
\hline Gender, N (\%) & & & & $<0.0001$ \\
\hline Male & $14,163(49.7)$ & $13,185(50.1)$ & $978(44.9)$ & \\
\hline Female & $14,325(50.3)$ & $13,126(49.9)$ & $1,199(55.1)$ & \\
\hline Race/Ethnicity, N (\%) & & & & 0.0004 \\
\hline White & $22,627(79.4)$ & $20,970(79.7)$ & $1,657(76.1)$ & \\
\hline Black & $3,371(11.8)$ & $3,049(11.6)$ & $322(14.8)$ & \\
\hline Hispanic & $589(2.1)$ & $538(2.0)$ & $51(2.3)$ & \\
\hline Asian & $1,236(4.3)$ & $1,134(4.3)$ & $102(4.7)$ & \\
\hline Native American & $92(0.3)$ & $83(0.3)$ & $9(0.4)$ & \\
\hline Other/unknown & $573(2.0)$ & $537(2.0)$ & $36(1.7)$ & \\
\hline Region, N (\%) & & & & 0.013 \\
\hline Northeast & $4,586(16.6)$ & $4,224(16.6)$ & $362(17.3)$ & \\
\hline Midwest & $6,400(23.2)$ & $5,958(23.3)$ & $442(21.1)$ & \\
\hline South & $11,405(41.3)$ & $10,486(41.1)$ & $919(44.0)$ & \\
\hline West & $5,227(18.9)$ & $4,859(19.0)$ & $368(17.6)$ & \\
\hline Charlson- Romano comorbidity Index Score & $3.61(3.22)$ & $3.56(3.20)$ & $4.27(3.39)$ & $<0.0001$ \\
\hline
\end{tabular}

${ }^{\mathrm{a}} \mathrm{No}$ allopurinol use in the baseline period of 365 days 


\section{Results}

\section{Study population characteristics}

There were a total of 28,488 episodes of incident allopurinol use (Table 1). Of these, 2,177 allopurinol use episodes were associated with incident stroke (7.6\% episodes; baseline period of 365 days without any stroke), while the majority did not (Fig. 1). Compared to incident allopurinol use without stroke, those with episodes of incident stroke on allopurinol were older, more likely to be women or white, and had higher Charlson index score (4.27 vs. 3.56 ); some differences were noted by region of residence (Table 1). Mean (median) length to first stroke event was
437 (median, 299) days and mean (median) follow-up time was 704 (median, 579) days.

Table 2 shows the crude incidence rate of stroke by allopurinol exposure. Crude incidence rates of stroke with and without allopurinol exposure were similar. Durations of allopurinol use longer than 6-month were associated with lower crude incidence rate of stroke compared to no allopurinol use (Table 2).

\section{Allopurinol and the risk of incident stroke}

Compared to non-use, age and Charlson comorbidity index were higher with allopurinol use for episodes with

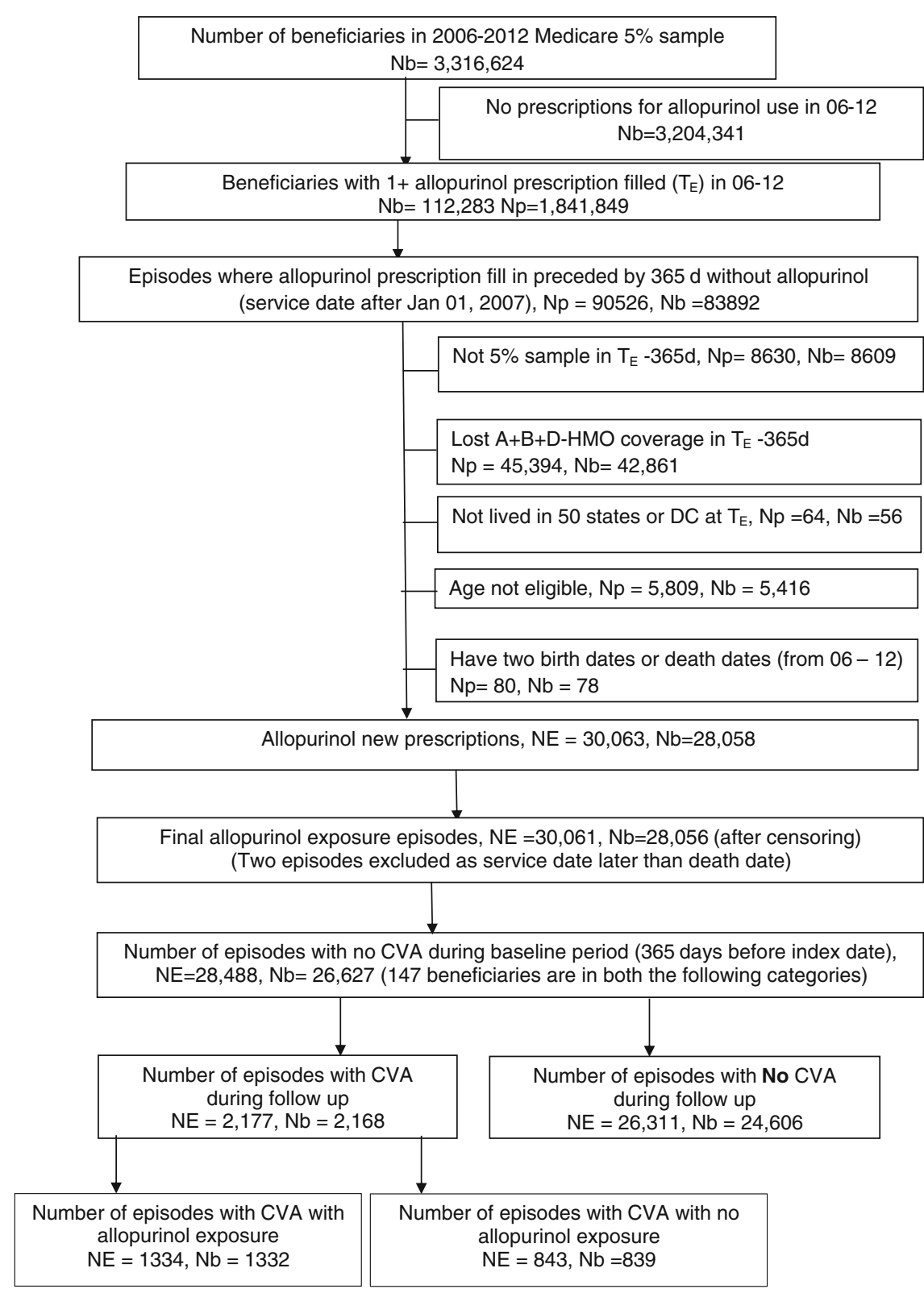

Fig. 1 Flow-chart of study cohort of incident allopurinol users from 2006-2012 for baseline of 365 days. Legend: NE, number of episodes; Nb, number of beneficiaries; Np, number of prescriptions 
Table 2 Crude incidence rate of CVA with allopurinol exposure and allopurinol use duration

\begin{tabular}{llll}
\hline & $\begin{array}{c}\text { Person-days } \\
\text { of follow up }\end{array}$ & $\begin{array}{l}\text { Stroke } \\
\text { cases }\end{array}$ & $\begin{array}{l}\text { Crude Stroke Incidence Rate } \\
\text { per 1,000,000 person-days }\end{array}$ \\
\hline $\begin{array}{l}\text { Allopurinol Exposure } \\
\text { Yes }\end{array}$ & & & \\
No & $12,900,000$ & 1,334 & 108 \\
Allopurinol use duration & $7,902,385$ & 843 & 106 \\
None & $7,902,385$ & 843 & 106 \\
1 to 180 days & $5,676,393$ & 728 & 128 \\
181 days to & $4,876,688$ & 476 & 98 \\
2 year & & & \\
>2 years & $1,721,507$ & 130 & 76
\end{tabular}

${ }^{a}$ Allopurinol exposure was defined as up to 30 days after last day of allopurinol prescription fill or refill to capture the biologic effect of allopurinol use

incident stroke (Table 3). In multivariable-adjusted analyses, allopurinol use was associated with $9 \%$ lower hazard ratio for stoke, 0.91 (95\% CI, 0.83 to 0.99). Age 75-< 85 and $\geq 85$, black race, higher Charlson-Romano index score and the use of beta-blockers were associated with higher hazards of incident stroke (Table 4). Specific hazards ratios by age, gender and race are shown in Additional file 1: Table S1.

We found that compared to no allopurinol use, allopurinol use durations of longer than 6-months were each associated with lower multivariable-adjusted hazard

Table 3 Demographic and comorbidity characteristics of episodes that ended in stroke by allopurinol use

\begin{tabular}{|c|c|c|c|}
\hline & \multicolumn{2}{|c|}{ Episodes with stroke } & \multirow[t]{2}{*}{$P$-value } \\
\hline & $\begin{array}{l}\text { Not on } \\
\text { Allopurinol } \\
\text { (NE = 843) }\end{array}$ & $\begin{array}{l}\text { On } \\
\text { Allopurinol } \\
(\mathrm{NE}=1,334)\end{array}$ & \\
\hline Age & $77.5(7.1)$ & $78.5(7.4)$ & 0.003 \\
\hline Gender & & & 0.95 \\
\hline Male & $378(44.8)$ & $600(45.0)$ & \\
\hline Female & $465(55.2)$ & $734(55.0)$ & \\
\hline Race & & & 0.54 \\
\hline White & $632(75.0)$ & $1,025(76.8)$ & \\
\hline Black & $128(15.2)$ & $194(14.5)$ & \\
\hline Others & $83(9.9)$ & $115(8.6)$ & \\
\hline $\begin{array}{l}\text { Charlson-Romano } \\
\text { Index Score }\end{array}$ & $3.99(3.27)$ & $4.44(3.44)$ & 0.002 \\
\hline \multicolumn{4}{|l|}{ Specific Comorbidities } \\
\hline Diabetes & $382(45.3)$ & $641(48.1)$ & 0.21 \\
\hline Hypertension & $724(85.9)$ & $1,174(88.0)$ & 0.15 \\
\hline Cardiovascular Disease & $128(15.2)$ & $232(17.4)$ & 0.18 \\
\hline Peripheral Vascular Disease & $187(22.2)$ & $302(22.6)$ & 0.80 \\
\hline Hyperlipidemia & $591(70.1)$ & $970(72.7)$ & 0.19 \\
\hline
\end{tabular}

NE number of episodes ratios for stroke: 181 days to 2 years, 0.88 (95\% CI, 0.78 to 0.99$)$ and $>2$ years, 0.79 (95\% CI, 0.65 to 0.96$)$ (Table 4).

Sensitivity analyses were performed adjusting for diabetes, hypertension, hyperlipidemia and tobacco use disorder as CAD risk factors, peripheral vascular disease and CAD confirmed the findings for both allopurinol use and the duration of allopurinol use (Additional file 2: Table S2).

\section{Subgroup analyses for allopurinol use duration and by the type of stroke}

In multivariable-adjusted subgroup analyses, allopurinol use durations of 181 days to 2 years and $>2$ years were associated with a reduction of hazard of stroke (Table 4), most evident for the age group 75-84, female gender and patients who were white (Fig. 2).

In multivariable-adjusted subgroup analyses, we found no significant association of allopurinol use or duration of allopurinol use with the risk of hemorrhagic stroke (Additional file 3: Table S3). On the other hand, significant associations were noted between allopurinol use and the risk of ischemic stroke, similar in magnitude to overall incident stroke analyses (Additional file 3: Table S3).

\section{Discussion}

We used an incident/new user design (new users of allopurinol indicated by a new allopurinol filled prescriptions) and a population-based approach to examine the effect of allopurinol use on the risk of stroke in the U.S. elderly. We controlled for patient demographics, comorbidity and use of cardio-protective medications to understand whether the association of allopurinol was independent of other CAD risk factors and treatments. This new knowledge is important, since the majority of strokes occur in adult Americans 65 years and older [29]. Subgroup analyses examined whether association of allopurinol was evident for separate analyses for ischemic and hemorrhagic stroke. Several study findings merit further discussion.

Our finding of independent association of incident allopurinol use with a reduction of hazard of incident stroke in the older adults compared to no allopurinol use, is novel. We are unaware of other studies that have examined this specific question. Most previous studies in this area of research had several limitations in that: (1) all studied a composite cardiovascular outcome that included heart failure, angina, MI etc. in addition to stroke [25-28]; (2) most used prevalent user design [26, 28]; and (3) all studies were in patient subpopulations with limited generalizability [25-28]. Composite outcome assessment did not allow the interpretation regarding the risk of stroke, due to inclusion of related outcomes, which might have different mechanisms than stroke, i.e., heart failure, angina etc. It is therefore not surprising that findings 
Table 4 Association of risk factors with incident stroke ${ }^{a}$ in patients who received allopurinol

\begin{tabular}{|c|c|c|c|c|c|c|}
\hline & \multicolumn{2}{|l|}{ Univariate } & \multicolumn{2}{|c|}{ Multivariable-adjusted (model 1)* } & \multicolumn{2}{|c|}{ Multivariable-adjusted (model 2)** } \\
\hline & $\mathrm{HR}(95 \% \mathrm{Cl})$ & $P$-value & $\mathrm{HR}(95 \% \mathrm{Cl})$ & $P$-value & $\mathrm{HR}(95 \% \mathrm{Cl})$ & $P$-value \\
\hline \multicolumn{7}{|l|}{ Age } \\
\hline $65-<75$ & Ref & & Ref & & Ref & \\
\hline $75-<85$ & $1.63(1.48,1.79)$ & $<0.0001$ & $1.54(1.40,1.70)$ & $<0.0001$ & $1.54(1.40,1.70)$ & $<0.0001$ \\
\hline$\geq 85$ & $2.09(1.86,2.35)$ & $<0.0001$ & $1.91(1.69,2.15)$ & $<0.0001$ & $1.91(1.69,2.16)$ & $<0.0001$ \\
\hline \multicolumn{7}{|l|}{ Gender } \\
\hline Male & Ref & & Ref & & Ref & \\
\hline Female & $1.21(1.12,1.32)$ & $<0.0001$ & $1.09(1.00,1.19)$ & 0.05 & $1.09(1.00,1.19)$ & 0.05 \\
\hline \multicolumn{7}{|l|}{ Race } \\
\hline White & Ref & & Ref & & Ref & \\
\hline Black & $1.40(1.24,1.57)$ & $<0.0001$ & $1.37(1.21,1.54)$ & $<0.0001$ & $1.36(1.21,1.53)$ & $<0.0001$ \\
\hline Other & $1.09(0.94,1.26)$ & 0.27 & $1.09(0.94,1.27)$ & 0.23 & $1.09(0.94,1.26)$ & 0.26 \\
\hline Charlson- Romano score & $1.11(1.10,1.12)$ & $<0.0001$ & $1.10(1.09,1.11)$ & $<0.0001$ & $1.10(1.09,1.11)$ & $<0.0001$ \\
\hline \multicolumn{7}{|c|}{ Cardiovascular drug use (Ref, no use) } \\
\hline Statins & $0.93(0.75,1.15)$ & 0.49 & $0.91(0.73,1.13)$ & 0.39 & $0.91(0.73,1.13)$ & 0.38 \\
\hline Beta blockers & $1.38(1.15,1.67)$ & 0.0007 & $1.40(1.15,1.70)$ & 0.0008 & $1.40(1.15,1.70)$ & 0.0008 \\
\hline Diuretics & $1.05(0.86,1.27)$ & 0.63 & $0.97(0.79,1.18)$ & 0.74 & $0.96(0.79,1.18)$ & 0.72 \\
\hline ACE inhibitor & $0.92(0.73,1.18)$ & 0.52 & $0.97(0.76,1.24)$ & 0.78 & $0.97(0.76,1.23)$ & 0.78 \\
\hline Allopurinol use (Ref, none) & $0.92(0.84,1.01)$ & 0.07 & $0.91(0.83,0.99)$ & 0.04 & - & - \\
\hline \multicolumn{7}{|l|}{ Allopurinol use duration } \\
\hline None & Ref & & & & Ref & \\
\hline $1-180$ days & $1.02(0.89,1.15)$ & 0.81 & & & $1.00(0.88,1.14)$ & 0.97 \\
\hline 181 days - 2 years & $0.90(0.80,1.01)$ & 0.07 & & & $0.88(0.78,0.99)$ & 0.03 \\
\hline$>2$ years & $0.78(0.64,0.96)$ & 0.02 & & & $0.79(0.65,0.96)$ & 0.02 \\
\hline
\end{tabular}

* Model 1 = Allopurinol use + age + race + gender + Charlson score + beta blockers + diuretics + ACE inhibitors + Statins

** Model 2 = Allopurinol use duration + age + race + gender + Charlson score + beta blockers + diuretics + ACE inhibitors + Statins

${ }^{a}$ No stroke within the baseline period of 365 days before the index date of allopurinol episode

ranged from a reduced risk of with allopurinol $[25,26]$ to an increased risk of composite cardiovascular outcome that included stroke $[27,28]$.

The stroke risk reduction associated with allopurinol use noted in our study and others may be related to several potential mechanisms. Allopurinol use was associated with attenuation of intercellular adhesion molecule1 levels in patients after a recent stroke [24]. Allopurinol use improved endothelial function and reduction of oxidative stress [14, 19-23] and reduced glycosylated hemoglobin [14]. Allopurinol reduced serum urate, and an elevated serum urate was associated with higher levels of chronic inflammatory markers in cardiac disease [37].

Regardless of the mechanism of action, the significant reduction of stroke risk associated with allopurinol has practical implications. The hazards of stroke were reduced by $9 \%$ for allopurinol use vs. non-use and reduced by $12-$ $21 \%$ with longer allopurinol use durations. Thus, the magnitudes of hazard reduction were moderate, not large.
Allopurinol is commonly used for the treatment of gout and reduced morbidity related to joint inflammation and pain, including disability, functional limitation and joint destruction. The potential added benefit of stroke reduction with allopurinol should be shared with patients with gout at the time of allopurinol treatment initiation. This discussion should impact the overall risk/benefit ratio of allopurinol, as well as serve as a motivating factor for improving allopurinol adherence and persistence, which is low for allopurinol in the first year after initiation [38]. More evidence is needed before an informed benefit/risk analysis of allopurinol use can be shared with patients with asymptomatic hyperuricemia without gout, where allopurinol use is currently not recommended [39].

Maximum hazard reduction in incident stroke with allopurinol use occurred in the $>6$ to 24 months and $>2$ years after new allopurinol use, with $12 \%$ and $21 \%$ hazard reduction, respectively. We noted an incremental stroke risk reduction associated with longer duration of allopurinol use. This important finding indicates 


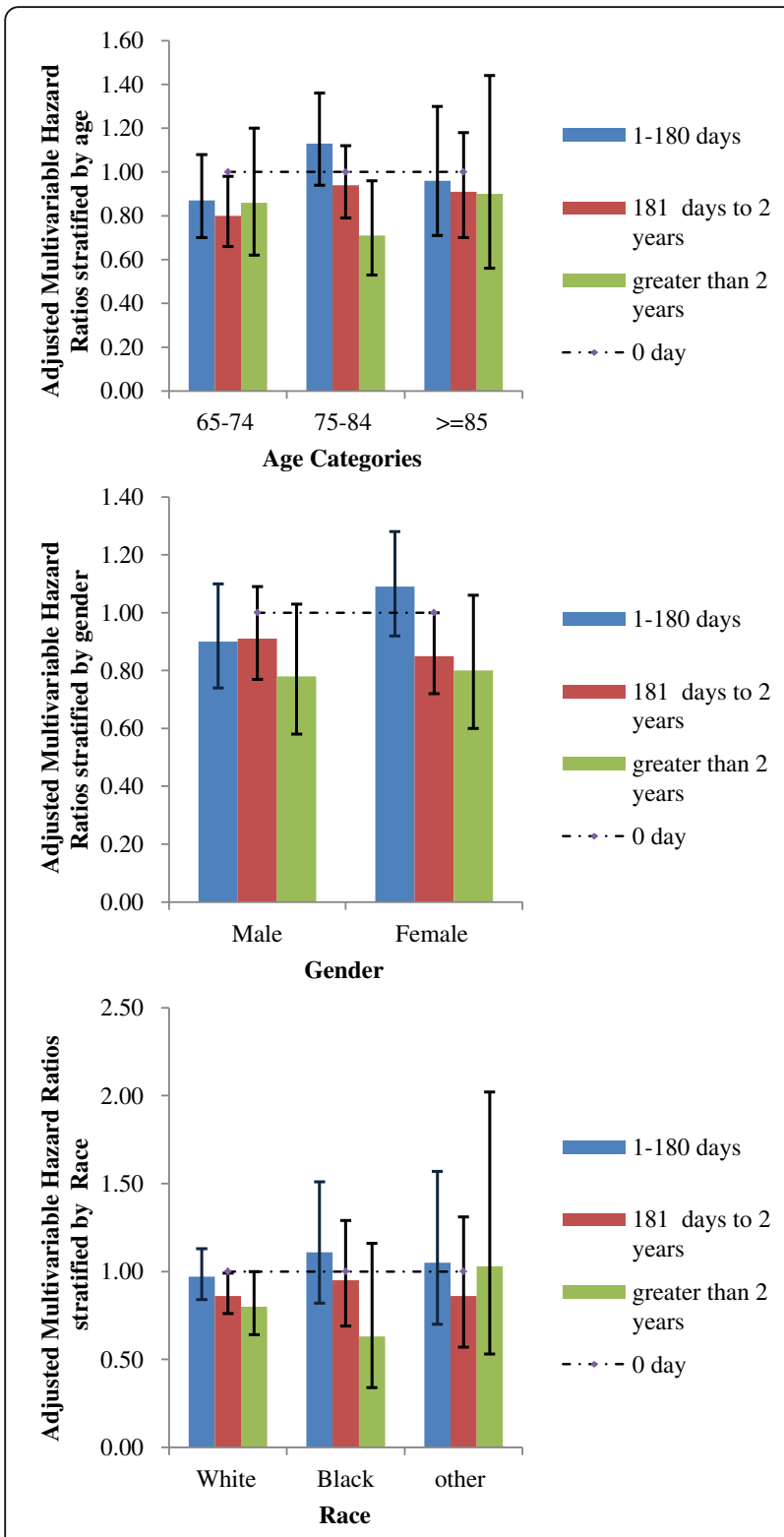

Fig. 2 Multivariable-adjusted* Hazard ratios of duration of allopurinol use with incident stroke by age (2a), gender (2b) and race (2c). Legend: *For the multivariable-adjusted subgroup analyses by age, gender and race, the main model was adjusted for all factors (age, gender, race and Charlson-Romano comrobidity score) except the factor of interest, respectively, which was used to perform stratified analysis (age, gender, race). We found that several subgroups had statistically significantly reduced hazard with allopurinol use, namely: Age group 65-75 years, 181 days to 2 years; Age group 75-84 years, $>2$ years; female gender, 181 days to 2 years; White race, 181 days to 2 years and $>2$ years. As expected, most of the subgroups had very few events, and therefore most subgroup analyses did not have power to detect significant differences within each subgroup

that allopurinol use is needed for $>6$ months to experience this potential benefit.

In exploratory multivariable-adjusted subgroup analyses, allopurinol use durations of 181 days to 2 years and $>2$ years were associated with a reduction of hazard of stroke, most evident for the age group 75-84, female gender and people who were white. Our study identified the sub- groups of patients likely to benefit the most with longer durations of allopurinol use. These findings need to be confirmed in future studies.

Our exploratory analyses added further clarity to this protective effect of allopurinol. Risk reduction associated with allopurinol use was noted for ischemic stroke, but not hemorrhagic stroke. These two types of stroke differ quite a bit in pathophysiology and outcomes [40]. It was reassuring that the protection for overall stroke risk is primarily due to protection against ischemic stroke, which is consistent with allopurinol's effect on oxidative stress, endothelial function and chronic inflammation [14, 19-23, 37].

Our study has several strengths and limitations. The diagnoses of CAD risk factors and Charlson-Romano index were based on the presence of ICD-9-CM codes, which may have led to misclassification bias. However, the codes for outcomes and covariates have reasonable accuracy [34-36], and the use of ICD-9-CM codes for Charlson-Romano index is a standard valid approach that is used commonly in analyses of large claims databases, such as Medicare. The observational study design does not allow us to eliminate confounder bias, due to unmeasured confounding; however, we included important demographic, clinical, risk factors and cardiac medications to account for important potential confounders. Serum urate levels were available for a small proportion of patients and therefore we could not examine whether the associations we noted were mediated through the reduction of serum urate or not. The database also did not allow us to include other variables such body mass index, diet and exercise, which might be potential risk factors for stroke.

The contribution of healthy adherer effect can not be ruled out, since patients adherent to/using allopurinol may have other healthy behaviors, such as high adherence to preventive care, other medications, healthier lifestyle etc., which may all reduce the risk of stroke etc. A filled prescription is a surrogate for, but can not be equated to the actual use of allopurinol. Since study's eligibility criteria included that patients to have continued coverage, these findings are likely not applicable to elderly who loose Medicare coverage. Whether these findings can be generalized to populations other than adults 65 years and older is not known, and further study is needed in this area.

Study strengths include a large sample size, the use of a sample that allows generalizable findings to Medicare enrollees, inclusion of $\mathrm{MI} /$ stroke risk factors in analyses to avoid confounding bias, and the robustness of estimates in sensitivity analyses. Implementation of new user design (i.e., examining only new users of allopurinol) in our study avoids over-adjustment for factors on the causal pathway, unlike the prevalent user design, used in previous studies. 


\section{Conclusions}

In conclusion, we found that allopurinol use was independently associated with a lower risk of incident stroke in older Americans. Allopurinol use for 6 months to 2 years and $>2$ years were associated with a lower risk of incident stroke. The stroke risk reduction associated with allopurinol was seen in the subgroup analysis for ischemic stroke, but not hemorrhagic stroke. Future studies need to explore the factors that mediate this potentially protective effect, and assess whether these factors are dependent or independent of serum urate reductions and anti-oxidant effects seen with allopurinol.

\section{Additional files}

Additional file 1: Table S1. Multivariable-adjusted* Hazard ratios of allopurinol use with incident stroke by age (a), gender (b) and race (c). (DOCX $79 \mathrm{~kb}$ )

Additional file 2: Table S2. Sensitivity analysis: Association of various risk factors with incident stroke* in patients who received allopurinol with cardiovascular disease and associated risk factors replacing Charlson score. (DOCX $161 \mathrm{~kb})$

Additional file 3: Table S3. Subgroup analyses by the type of stroke. (DOCX 89 kb)

\section{Acknowledgements}

None.

\section{Funding}

This material is the result of work supported by research funds from the Division of Rheumatology at the University of Alabama at Birmingham and the resources and use of facilities at the Birmingham VA Medical Center, Birmingham, Alabama, USA. The funding body did not play any role in design, in the collection, analysis, and interpretation of data; in the writing of the manuscript; and in the decision to submit the manuscript for publication.

\section{Availability of data and materials}

These data are in the public domain for anyone to use and analyze and are available from the Agency for Healthcare Research and Quality (AHRQ).

\section{Authors' contributions}

JAS designed the study, developed study protocol, reviewed analyses and wrote the first draft of the paper. SY performed the data abstraction and data analyses. All authors made revisions to the manuscript and read and approved the final manuscript

\section{Competing interests}

There are no financial conflicts related directly to this study. JAS has received research grants from Takeda and Savient and consultant fees from Savient, Takeda, Regeneron, Merz, Bioiberica, Crealta and Allergan pharmaceuticals, WebMD, UBM LLC and the American College of Rheumatology. JAS serves as the principal investigator for an investigator-initiated study funded by Horizon pharmaceuticals through a grant to DINORA, Inc., a 501 (c) (3) entity. JAS is a member of the executive of OMERACT, an organization that develops outcome measures in rheumatology and receives arms-length funding from 36 companies; a member of the American College of Rheumatology's (ACR) Annual Meeting Planning Committee (AMPC); Chair of the ACR Meet-the-Professor, Workshop and Study Group Subcommittee; and a member of the Veterans Affairs Rheumatology Field Advisory Committee. SY has no conflicts to declare. There are no nonfinancial competing interests for any of the authors.

\section{Consent for publication}

No individual person's data were presented in any form in this study and therefore no consent to publish is required.

\section{Ethics approval and consent to participate}

The University of Alabama at Birmingham's Institutional Review Board approved this study and all investigations were conducted in conformity with ethical principles of research. The IRB waived the need for informed consent for this analysis of publically available data.

\section{Author details}

${ }^{1}$ Medicine Service, Birmingham VA Medical Center, Birmingham, AL, USA. ${ }^{2}$ Department of Medicine at School of Medicine, University of Alabama at Birmingham (UAB), Birmingham, AL, USA. ${ }^{3}$ Division of Epidemiology at School of Public Health, University of Alabama at Birmingham (UAB), Birmingham, AL, USA. ${ }^{4}$ Department of Orthopedic Surgery, Mayo Clinic College of Medicine, Rochester, MN, USA. ${ }^{5}$ University of Alabama, Faculty Office Tower 805B, 510 20th Street S, Birmingham, AL 35294, USA.

Received: 26 April 2016 Accepted: 29 August 2016

Published online: 07 September 2016

\section{References}

1. Murphy SL, Xu JQ, Kochanek KD: Deaths: Final data for 2010. National vital statistics reports; vol 61 no 4. Hyattsville, MD: National Center for Health Statistics. 2013. 2013

2. Feigin $\mathrm{VL}$, Forouzanfar $M \mathrm{H}$, Krishnamurthi $\mathrm{R}$, Mensah GA, Connor M, Bennett DA, Moran AE, Sacco RL, Anderson L, Truelsen T, et al. Global and regional burden of stroke during 1990-2010: findings from the global burden of disease study 2010. Lancet. 2014;383(9913):245-54.

3. Blackwell DL, Lucas JW, Clarke TC: Summary health statistics for U.S. adults. National Health Interview Survey, 2012. National Center for Health Statistics. Vital Health Stat. 2014;(260):1-161.

4. Kim K, Kim YM, Kim EK. Correlation between the activities of daily living of stroke patients in a community setting and their quality of life. J Phys Ther Sci. 2014;26(3):417-9.

5. Dhamoon MS, McClure LA, White $\mathrm{CL}$, Lau H, Benavente O, Elkind MS. Quality of life after lacunar stroke: the secondary prevention of small subcortical strokes study. J Stroke Cerebrovasc Dis. 2014:23(5):1131-7.

6. van Mierlo ML, van Heugten CM, Post MW, Lindeman E, de Kort PL, VisserMeily JM. A longitudinal cohort study on quality of life in stroke patients and their partners: Restore4Stroke Cohort. Int J Stroke. 2014;9(1):148-54.

7. Sturm JW, Dewey HM, Donnan GA, Macdonell RA, McNeil JJ, Thrift AG. Handicap after stroke: how does it relate to disability, perception of recovery, and stroke subtype?: the north North East Melbourne Stroke Incidence Study (NEMESIS). Stroke. 2002;33(3):762-8.

8. Broussalis E, Killer M, McCoy M, Harrer A, Trinka E, Kraus J. Current therapies in ischemic stroke. Part A. Recent developments in acute stroke treatment and in stroke prevention. Drug Discov Today. 2012;17(7-8):296-309.

9. Sarawate CA, Brewer KK, Yang W, Patel PA, Schumacher HR, Saag KG, Bakst AW. Gout medication treatment patterns and adherence to standards of care from a managed care perspective. Mayo Clin Proc. 2006:81(7):925-34.

10. Singh JA, Hodges JS, Asch SM. Opportunities for improving medication use and monitoring in gout. Ann Rheum Dis. 2009:68(8):1265-70.

11. Bayram D, Tugrul Sezer M, Inal S, Altuntas A, Kidir V, Orhan H. The effects of allopurinol on metabolic acidosis and endothelial functions in chronic kidney disease patients. Clin Exp Nephrol. 2015;19(3):443-9.

12. Butler R, Morris AD, Belch JJ, Hill A, Struthers AD. Allopurinol normalizes endothelial dysfunction in type 2 diabetics with mild hypertension. Hypertension. 2000;35(3):746-51.

13. Doehner W, Schoene N, Rauchhaus M, Leyva-Leon F, Pavitt DV, Reaveley DA, Schuler G, Coats AJ, Anker SD, Hambrecht R. Effects of xanthine oxidase inhibition with allopurinol on endothelial function and peripheral blood flow in hyperuricemic patients with chronic heart failure: results from 2 placebo-controlled studies. Circulation. 2002;105(22):2619-24.

14. Dogan A, Yarlioglues M, Kaya MG, Karadag Z, Dogan S, Ardic I, Dogdu O, Kilinc $Y$, Zencir C, Akpek $M$, et al. Effect of long-term and high-dose allopurinol therapy on endothelial function in normotensive diabetic patients. Blood Press. 2011;20(3):182-7.

15. El Solh AA, Saliba R, Bosinski T, Grant BJ, Berbary E, Miller N. Allopurinol improves endothelial function in sleep apnoea: a randomised controlled study. Eur Respir J. 2006:27(5):997-1002.

16. Noman A, Ang DS, Ogston S, Lang CC, Struthers AD. Effect of high-dose allopurinol on exercise in patients with chronic stable angina: a randomised placebo controlled crossover trial. Lancet. 2010;375(9732):2161-7. 
17. Szwejkowski BR, Gandy SJ, Rekhraj S, Houston JG, Lang CC, Morris AD, George J, Struthers AD. Allopurinol reduces left ventricular mass in patients with type 2 diabetes and left ventricular hypertrophy. J Am Coll Cardiol. 2013;62(24):2284-93.

18. Rekhraj S, Gandy SJ, Szwejkowski BR, Nadir MA, Noman A, Houston JG, Lang CC, George J, Struthers AD. High-dose allopurinol reduces left ventricular mass in patients with ischemic heart disease. J Am Coll Cardiol. 2013;61(9): 926-32.

19. Kanbay M, Huddam B, Azak A, Solak Y, Kadioglu GK, Kirbas I, Duranay M, Covic A, Johnson RJ. A randomized study of allopurinol on endothelial function and estimated glomular filtration rate in asymptomatic hyperuricemic subjects with normal renal function. Clin J Am Soc Nephrol. 2011;6(8):1887-94.

20. Yiginer O, Ozcelik F, Inanc T, Aparci M, Ozmen N, Cingozbay BY, Kardesoglu E, Suleymanoglu S, Sener G, Cebeci BS. Allopurinol improves endothelial function and reduces oxidant-inflammatory enzyme of myeloperoxidase in metabolic syndrome. Clin Res Cardiol. 2008:97(5):334-40.

21. Kanbay M, Siriopol D, Nistor I, Elcioglu OC, Telci O, Takir M, Johnson RJ, Covic A. Effects of allopurinol on endothelial dysfunction: a meta-analysis. Am J Nephrol. 2014;39(4):348-56.

22. Melendez-Ramirez G, Perez-Mendez O, Lopez-Osorio C, Kuri-Alfaro J, EspinolaZavaleta N. Effect of the treatment with allopurinol on the endothelial function in patients with hyperuricemia. Endocr Res. 2012;37(1):1-6.

23. Kao MP, Ang DS, Gandy SJ, Nadir MA, Houston JG, Lang CC, Struthers AD. Allopurinol benefits left ventricular mass and endothelial dysfunction in chronic kidney disease. J Am Soc Nephrol. 2011;22(7):1382-9.

24. Muir SW, Harrow C, Dawson J, Lees KR, Weir CJ, Sattar N, Walters MR. Allopurinol use yields potentially beneficial effects on inflammatory indices in those with recent ischemic stroke: a randomized, double-blind, placebocontrolled trial. Stroke. 2008;39(12):3303-7.

25. Goicoechea M, Garcia De Vinuesa S, Verdalles U, Verde E, Macias N, Santos A, Perez De Jose A, Cedeno S, Linares T, Luno J. Allopurinol and progression of CKD and cardiovascular events: long-term follow-up of a randomized clinical trial. Am J Kidney Dis. 2015;65(4):543-9.

26. Terawaki H, Nakayama M, Miyazawa E, Murata Y, Nakayama K, Matsushima M, Miyazaki M, Sato H, Sato M, Sato T, et al. Effect of allopurinol on cardiovascular incidence among hypertensive nephropathy patients: the Gonryo study. Clin Exp Nephrol. 2013;17(4):549-53.

27. Wei L, Mackenzie IS, Chen Y, Struthers AD, MacDonald TM. Impact of allopurinol use on urate concentration and cardiovascular outcome. $\mathrm{Br}$ J Clin Pharmacol. 2011;71(4):600-7.

28. Kok VC, Horng JT, Chang WS, Hong YF, Chang TH. Allopurinol therapy in gout patients does not associate with beneficial cardiovascular outcomes: a population-based matched-cohort study. PLoS One. 2014;9(6):e99102.

29. Hall MJLS, DeFrances CJ. Hospitalization for stroke in U.S. hospitals, 19892009, NCHS data brief, No. 95. Hyattsville: National Center for Health Statistics; 2012.

30. Charlson ME, Pompei P, Ales KL, MacKenzie CR. A new method of classifying prognostic comorbidity in longitudinal studies: development and validation. J Chronic Dis. 1987;40(5):373-83.

31. Romano PS, Roos LL, Jollis JG. Adapting a clinical comorbidity index for use with ICD-9-CM administrative data: differing perspectives. J Clin Epidemiol. 1993:46(10):1075-9. discussion 1081-1090.

32. Chu YT, Ng YY, Wu SC. Comparison of different comorbidity measures for use with administrative data in predicting short- and long-term mortality. BMC Health Serv Res. 2010;10:140.

33. Yurkovich M, Avina-Zubieta JA, Thomas J, Gorenchtein M, Lacaille D. A systematic review identifies valid comorbidity indices derived from administrative health data. J Clin Epidemiol. 2015;68(1):3-14.

34. Kiyota Y, Schneeweiss S, Glynn RJ, Cannuscio CC, Avorn J, Solomon DH. Accuracy of Medicare claims-based diagnosis of acute myocardial infarction: estimating positive predictive value on the basis of review of hospital records. Am Heart J. 2004;148(1):99-104

35. Birman-Deych E, Waterman AD, Yan Y, Nilasena DS, Radford MJ, Gage BF. Accuracy of ICD-9-CM codes for identifying cardiovascular and stroke risk factors. Med Care. 2005;43(5):480-5.

36. Thigpen JL, Dillon C, Forster KB, Henault L, Quinn EK, Tripodis Y, Berger PB, Hylek EM, Limdi NA. Validity of international classification of disease codes to identify ischemic stroke and intracranial hemorrhage among individuals with associated diagnosis of atrial fibrillation. Circ Cardiovasc Qual Outcomes. 2015;8(1):8-14.
37. Leyva F, Anker SD, Godsland IF, Teixeira M, Hellewell PG, Kox WJ, PooleWilson PA, Coats AJ. Uric acid in chronic heart failure: a marker of chronic inflammation. Eur Heart J. 1998;19(12):1814-22.

38. Briesacher BA, Andrade SE, Fouayzi H, Chan KA. Comparison of drug adherence rates among patients with seven different medical conditions. Pharmacotherapy. 2008;28(4):437-43.

39. Khanna D, Fitzgerald JD, Khanna PP, Bae S, Singh MK, Neogi T, Pillinger MH, Merill J, Lee S, Prakash S, et al. American College of Rheumatology guidelines for management of gout. Part 1: systematic nonpharmacologic and pharmacologic therapeutic approaches to hyperuricemia. Arthritis Care Res (Hoboken). 2012;64(10):1431-46. 2012.

40. Paolucci S, Antonucci G, Grasso MG, Bragoni M, Coiro P, De Angelis D, Fusco FR, Morelli D, Venturiero V, Troisi E, et al. Functional outcome of ischemic and hemorrhagic stroke patients after inpatient rehabilitation: a matched comparison. Stroke. 2003:34(12):2861-5.

\section{Submit your next manuscript to BioMed Central and we will help you at every step:}

- We accept pre-submission inquiries

- Our selector tool helps you to find the most relevant journal

- We provide round the clock customer support

- Convenient online submission

- Thorough peer review

- Inclusion in PubMed and all major indexing services

- Maximum visibility for your research

Submit your manuscript at www.biomedcentral.com/submit
Biomed Central 